\title{
Basic Considerations for Container Closure Selection of Parenteral Drug Products
}

\author{
Lakshmi Prasanna Kolluru*
}

Medefil Inc, Glendale Heights, IL 60139, USA.

*Corresponding author: Lakshmi Prasanna Kolluru, Sr. Formulation Scientist, Medefil Inc., 250/405 Windy Point Drive, Glendale Heights, IL 60139, USA, Tel: +1-630-682-4600; Email: Prasanna.Kolluru@medefilinc.com

Received date: May 02, 2017; Accepted date: May 10, 2017; Published date: May 16, 2017

Copyright: () 2017 Kolluru LP. This is an open-access article distributed under the terms of the Creative Commons Attribution License, which permits unrestricted use, distribution, and reproduction in any medium, provided the original author and source are credited.

\section{Editorial}

By definition, container closure system encompasses all components of the packaging system that hold and protect the drug product. It includes primary packaging system (components with which the drug product comes in direct contact) and secondary packaging system (components that offer additional protection to the system but do not come in direct contact with the drug product). This article focuses on primary packaging components as they have a significant effect on the critical quality attributes of the drug product throughout the product shelf-life. Selection of an appropriate container closure system for a parenteral drug product is of utmost importance as Food and Drug Administration (FDA) classified that the probability of interaction of the drug product with the packaging component is high and the risks associated from such interactions are highest for injections administered via parenteral route [1]. Impeachment of container closure integrity leads to compromised sterility of the drug product system, which in turn is one of the major reasons for FDA drug product recalls in 2016 [2].

Various factors including characteristics of the drug product, intended use and compatibility with manufacturing equipment and other packaging components have to be taken into consideration in selection of container closure system for a parenteral drug product with acceptable quality and sterility.

Characteristics of the drug product: Stability and Sensitivity of the drug product are crucial parameters to be considered in selecting appropriate container closure components for the drug product. If the drug product is not adequately buffered and $\mathrm{pH}$ fluctuations are common over a period of time, ammonium sulphate treated vials should be preferred over untreated glass vials.

Similarly, if the drug product contains a chemical or organic solvent that has tendency to interact with stopper leading to loss of assay or presence of extractable/leachable, fluorotec coated stoppers are preferred to reduce the drug product-stopper interaction.

In addition, for drug products that cannot be sterilized by terminal sterilization and hence manufactured by aseptic processing, all the container closure components of the system have to be individually sterilized, depyrogenated and monitored for bio burden before use with drug product [3]. For example, a liquid drug product to be filled in vials should have all the glass vials, stoppers, filters and tubings with which the drug product comes in contact with washed, depyrogenated and sterilized (with a method suitable for the component under study). When the manufacturing facility does not have facilities for depyrogenation and sterilizing the components, Ready-To-Use (RTU) closure components can be employed in manufacturing of the drug product. In addition to ease of operation, studies have shown that usage of RTU components can aid in reducing the risk on contamination of the drug product [4].

\section{Intended use}

Container-closure selection varies for liquid and lyophilized drug products. Traditionally, tubular vials are preferred over molded vials during lyophilization because of the flat bottom of vials that help in uniform heat transfer from the freeze drying shelves to the drug product. As the containers require partial stoppering during lyophilization, stoppers used also vary from general liquid products.

\section{Machinability}

Components of container closure system should be compatible with the manufacturing equipment for viable commercialization of the product. Components that have high risk of interaction with manufacturing parts are suitably processed to enhance their machinability. For instance, stoppers with silicone coating are employed to reduce friction with manufacturing parts and aid in efficient sealing of the container system.

Interaction with other component systems: Compatibility with other component systems is a major consideration in selecting container closure components. Blowback designs of vials and stoppers (No blow back, American blowback or European blowback) have to be considered to ensure effective sealability of selected vial and stopper combination. Besides analyzing product diagrams, engaging vial and stopper manufacturers in the decision making process helps in selecting right combination for the drug product.

Finally, evaluating the finalized container closure system of the drug product for safety is of critical importance. Various physico-chemical and biological tests are conducted to ensure safety of the drug product system [5-8]. The requirements are more stringent for biological parenteral products, for which containers used for storage or shipping of bulk drug product should also be studied for suitability in detail [9].

In conclusion, container closure integrity is a critical quality attribute of parenteral drug products. Hence, container closure components have to be selected with careful consideration and extensive evaluation of the drug product and manufacturing attributes.

\section{References}

1. Food and Drug Administration (FDA) (1999) Guidance for industry, container and closure systems for packaging. Human Drugs and Biologics.

2. Lakshmi Prasanna K (2017) Sterility Assurance of parenteral productsmajor deficiency for recall. J Pharmacovigil 5: e168. 
Citation: Kolluru LP (2017) Basic Considerations for Container Closure Selection of Parenteral Drug Products . Pharm Anal Acta 8: e189. doi:

Page 2 of 2

3. FDA (2003) Guidance for industry, sterile drug products produced by aseptic processing-current good manufacturing practices.

4. Curry W, Conway S, Goodfield C, Miller K, Mueller R, et al (2010) Reducing the Risk of contamination of sterile parenteral products via ready-to-use closure components. AAPS PharmSciTech 11: 1572-1579.

5. http://www.pharmacopeia.cn/v29240/usp29nf24s0_c1207.html

6. http://www.usp.org/sites/default/files/usp_pdf/EN/USPNF/iras/gc-87biolgoical-reactivity.pdf
7. http://www.pharmacopeia.cn/v29240/usp29nf24s0_c88.html

8. http://www.usp.org/sites/default/files/usp_pdf/EN/USPNF/ generalChapter381.pdf

9. Food and Drug Administration (FDA) (2002) Guidance for industry, container and closure systems for packaging human drugs and biologicsquestions and answers, deregulated wnt $/ \beta$-catenin signaling. BMC Genomics 15: 74 . 